\title{
Outcomes with a shorter multidrug- resistant tuberculosis regimen from Karakalpakstan, Uzbekistan
}

\author{
Philipp du $\operatorname{Cros}^{1,2}$, Atadjan Khamraev ${ }^{3}$, Zinaida Tigay ${ }^{4}$, Tleubergen Abdrasuliev ${ }^{5}$, \\ Jane Greig ${ }^{1,2}$, Graham Cooke ${ }^{6}$, Krzysztof Herboczek$^{1}$, Tanya Pylypenko ${ }^{5}$, \\ Catherine Berry ${ }^{1}$, Amrita Ronnachit $^{5}$, David Lister ${ }^{5}$, Sebastian Dietrich ${ }^{7}$, \\ Cono Ariti ${ }^{8}$, Khasan Safaev $^{9}$, Bern-Thomas Nyang'wa ${ }^{1}$, Nargiza Parpieva ${ }^{9}$, \\ Mirzagalib Tillashaikhov ${ }^{9}$ and Jay Achar (10 ${ }^{1,10}$
}

\section{ABSTRACT}

Background: In 2016, World Health Organization guidelines conditionally recommended standardised shorter 9-12-month regimens for multidrug-resistant (MDR) tuberculosis (TB) treatment. We conducted a prospective study of a shorter standardised MDR-TB regimen in Karakalpakstan, Uzbekistan.

Methods: Consecutive adults and children with confirmed rifampicin-resistant pulmonary TB were enrolled between September 1, 2013 and March 31, 2015; exclusions included prior treatment with second-line anti-TB drugs, and documented resistance to ofloxacin or to two second-line injectable agents. The primary outcome was recurrence-free cure at 1 year following treatment completion.

Results: Of 146 enrolled patients, 128 were included: 67 female (52.3\%), median age 30.1 (interquartile range 23.8-44.4) years. At the end of treatment, $71.9 \%$ (92 out of 128) of patients achieved treatment success, with $68 \%$ (87 out of 128) achieving recurrence-free cure at 1 year following completion. Unsuccessful outcomes during treatment included $22(17.2 \%)$ treatment failures with fluoroquinolone-resistance amplification in 8 patients (8 out of 22, 36.4\%); 12 (9.4\%) lost to follow-up; and 2 (1.5\%) deaths. Recurrence occurred in one patient. Fourteen patients (10.9\%) experienced serious adverse events. Baseline resistance to both pyrazinamide and ethambutol (adjusted OR 6.13, 95\% CI 2.01; 18.63) and adherence <95\% (adjusted OR $5.33,95 \%$ CI 1.73 ; 16.36) were associated with unsuccessful outcome in multivariable logistic regression.

Conclusions: Overall success with a standardised shorter MDR-TB regimen was moderate with considerable treatment failure and amplification of fluoroquinolone resistance. When introducing standardised shorter regimens, baseline drug susceptibility testing and minimising missed doses are critical. High rates globally of pyrazinamide, ethambutol and ethionamide resistance raise questions of continued inclusion of these drugs in shorter regimens in the absence of drug susceptibility testing-confirmed susceptibility.

$@$ ERSpublications

A standardised shorter MDR-TB regimen observational study in Uzbekistan showed moderate success, but high treatment failure with significant risk of amplification of fluoroquinolone resistance https://bit.ly/3o8vfJz

Cite this article as: du Cros P, Khamraev A, Tigay Z, et al. Outcomes with a shorter multidrugresistant tuberculosis regimen from Karakalpakstan, Uzbekistan. ERJ Open Res 2021; 7: 00537-2020 [https://doi.org/10.1183/23120541.00537-2020].

This article has supplementary material available from openres.ersjournals.com.

This study is registered at www.clinicaltrials.gov with identifier number NCT02496572. Data sharing is available in accordance with the MSF data sharing policy available at the MSF Field Research Website (https:/fieldresearch.msf.org/ bitstream/handle/10144/306501/MSF+data+sharing+policy+final+061213.pdf?sequence=1). The policy states, "MSF, as an international medical humanitarian organisation, and Epicentre, its Research affiliate, are committed to share and disseminate health data from their programs and Research in an open, timely and transparent manner in order to promote health benefits for populations while respecting ethical and legal obligations, notably towards MSF patients, Research participants and their communities".

Received: 29 July 2020 | Accepted: 12 Oct 2020

Copyright $\odot$ ERS 2021. This article is open access and distributed under the terms of the Creative Commons Attribution Non-Commercial Licence 4.0. 


\section{Introduction}

Rifampicin-resistant (RR) and multidrug-resistant (MDR) tuberculosis (TB) remain a public health emergency, with an estimated 484000 cases occurring worldwide in 2018 [1]. Diagnosis and management are expensive and resource-intensive, with only $32 \%$ of the estimated globally incident MDR-TB cases able to access treatment to international standards [1]. Standard treatment is characterised by high rates of adverse events, prolonged duration, and success rates that can be as low as $60 \%$ [2]. In 2010, a single-arm cohort study in Bangladesh assessed treatment using an initial 4-6-month intensive phase with seven anti-TB drugs, followed by 5 months with four drugs, and reported $88 \%$ treatment success, with low relapse risk [3]. The STREAM trial, a phase III randomised study, demonstrated noninferiority of a similar standardised shorter regimen to long regimens recommended in World Health Organization (WHO) 2011 guidelines [4]. In 2016, WHO guidelines conditionally recommended a shorter 9-12-month MDR-TB regimen for patients meeting specific criteria, based on results of a systematic review and individual patient data meta-analysis $[5,6]$. However, uncertainty remains about the regimen's effectiveness in the presence of resistance to constituent drugs, including fluoroquinolones, ethambutol, pyrazinamide and prothionamide [7-11].

Uzbekistan is a Central Asian country of more than 34 million people. It is estimated that 4700 people suffer with RR/MDR-TB each year in the country, with $15 \%$ of new cases estimated to display RR [1]. Amongst people suffering with TB in Central Asia, prevalence of RR and second-line drug resistance is high [1]. Médecins Sans Frontières (MSF) has been supporting the Ministry of Health to treat RR/ MDR-TB in the Republic of Karakalpakstan, Uzbekistan, since 2003. High numbers of patients, moderate rates of success and high loss to follow-up, despite adherence to international standards, prompted investigation of innovative treatment approaches [12]. In 2013, we initiated a prospective cohort study investigating the effectiveness of a standardised shorter regimen (SSR) for treatment of RR/MDR-TB, in accordance with WHO's recommendations on operational research [13]. We hypothesised that a SSR is effective under routine programme conditions in settings with high prevalence of RR/MDR-TB and second-line drug resistance.

\section{Methods}

Study design, setting, participants

We carried out a prospective observational cohort study enrolling RR/MDR-TB patients between September 1, 2013 and March 31, 2015 in the Republic of Karakalpakstan, Uzbekistan [14]. Enrolment occurred in three districts of the country, namely Shumunay, Kegeily and Nukus City. The primary outcome was recurrence-free cure, 1 year following treatment completion. Secondary outcomes were the frequency of all adverse events, risk factors for unsuccessful outcomes (death, loss to follow-up (LTFU), treatment failure, recurrence), and the frequency of resistance amplification.

All consecutive patients with pulmonary $\mathrm{TB}$ and newly identified RR who did not have a history of prior treatment with second-line anti-TB drugs for more than 1 month, and for whom informed consent could be obtained, were eligible for inclusion. Children suspected as having MDR-TB, based on contact history with a source case with confirmed RR-TB, were also eligible. Exclusion criteria comprised: baseline contraindications to study medications, estimated creatinine clearance of $<30 \mathrm{~mL} \cdot \mathrm{min}^{-1}$ using the Cockcroft-Gault equation, resistance to ofloxacin or two second-line injectables (kanamycin and capreomycin), extrapulmonary TB without lung involvement, osteoarticular or meningeal TB, critical illness and in the judgement of the treating physician unlikely to survive more than 1 week, and Fridericia-adjusted QT interval $>500 \mathrm{~ms}$ [15-17]. Patients starting treatment based on genotypic drug susceptibility testing (DST) who subsequently met any exclusion criteria from phenotypic DST, were withdrawn and commenced on individualised treatment per WHO recommendations.

The treatment regimen consisted of seven drugs in the intensive phase: pyrazinamide (Z), ethambutol (E), high-dose isoniazid $(\mathrm{H})$, moxifloxacin $(\mathrm{Mfx})$, capreomycin $(\mathrm{Cm})$ or kanamycin $(\mathrm{Km})$, prothionamide (Pto) and clofazimine $(\mathrm{Cfz})$ for 4 to 6 months. This was followed by a fixed 5-month continuation phase with Z, E, Mfx, Pto and Cfz, after documentation of sputum-smear microscopy conversion and at least one negative

\footnotetext{
Affiliations: ${ }^{1}$ Manson Unit, Médecins Sans Frontières, London, UK. ${ }^{2}$ Burnet Institute, Melbourne, Australia. ${ }^{3}$ Supreme Council of Karakalpakstan, Nukus, Karakalpakstan. ${ }^{4}$ Ministry of Health, Nukus, Karakalpakstan. ${ }^{5}$ Médecins Sans Frontières, Nukus, Uzbekistan. ${ }^{6}$ Imperial College London, London, UK. ${ }^{7}$ Médecins Sans Frontières, Berlin, Germany. ${ }^{8}$ Cardiff University School of Medicine, Cardiff, UK. 9 Specialized Scientific Practical Medical Center of Phthisiology and Pulmonology, Tashkent, Uzbekistan. ${ }^{10}$ Karolinska Institutet, Stockholm, Sweden.
}

Correspondence: Philipp du Cros, Burnet Institute, 85 Commercial Road, Melbourne, Victoria 3000, Australia. E-mail: philipp.ducrosaburnet.edu.au 
culture. Dosing was weight-based (supplementary appendix) and all treatment was provided under routine programmatic conditions, cost-free to the patient. Trained nurses provided directly observed therapy (DOT) in the community, 7 days per week, either at outpatient clinics or the patient's home. All patients with severe clinical conditions were hospitalised for treatment. Support for treatment adherence was provided according to national recommendations: a nurse educator provided treatment education, nutritional support for low body mass index (BMI), and psychosocial support and counselling. Adverse event monitoring was performed systematically, with daily nurse and monthly physician assessment and attention to appropriate management of side effects including use of ECG and audiometry (detailed description of the study monitoring and management of adverse events is included in the study protocol [14]). Adverse event grading was performed according to the Division of AIDS Table for Grading the Severity of Adverse Events [18]. Laboratory diagnosis was centralised and utilised Xpert MTB/RIF (Cepheid, Sunnyvale, CA, USA), Genotype MTBDRplus (Hain Lifescience, Nehren, Germany), Genotype MTBDRsl version 1 (Hain Lifescience, Nehren, Germany) and MGIT960 (Becton Dickinson Diagnostic System, Sparks, MD) to confirm eligibility, culture status and drug susceptibility. DST testing was performed using BACTEC ${ }^{\text {TM }}$ MGIT960 SIRE kit and the BACTEC $^{\text {TM }}$ MGIT960 PZA kit (Becton Dickinson Diagnostic System, Sparks, MD, USA) and published second-line DST methods and critical concentrations $[19,20]$. The study reported to an independent data safety and monitoring board.

\section{Definitions}

Baseline smear, culture status and DST were defined by the availability of results from specimens submitted from 90 days prior and up to 7 days after treatment initiation. Results for baseline smear and culture were taken from the specimen submitted closest to treatment initiation. Baseline DST was defined by the most resistant result from specimens submitted during the eligible period. Patients with molecular DST test results indicating RR and fluoroquinolone sensitivity, but without phenotypic DST results during the defined baseline period, were continued on treatment and included in the analysis.

Determination of the end of treatment outcome followed WHO definitions for MDR-TB programmes, adapted for changes relevant to the shortened duration of treatment (supplementary appendix; [21]). A the end of treatment, successful outcomes were defined as cure or treatment completion, whereas LTFU, death and treatment failure were considered as unsuccessful. Whole-genome sequencing of isolates was not available, so differentiating relapse from re-infection was not possible. Recurrence was defined as any successfully treated patient who was culture-positive for RR-TB during 12 months of post-treatment follow-up.

\section{Data collection and analysis}

Data on age, sex, BMI, employment status and treatment adherence were collected for all patients. Treatment adherence assessed by DOT was determined cumulatively, based on the number of days of complete TB treatment taken, divided by the number of days of prescribed TB treatment. For patients who were LTFU, days of prescribed treatment was calculated until the last day of observed treatment. Treatment adherence $<95 \%$ was classified as poor, since treatment was directly observed. Measuring treatment adherence, diabetes mellitus screening using random and fasting blood sugar levels, and screening for HIV were all mandatory in the TB programme. Baseline chest radiographs were reviewed for the presence of cavities. We estimated that 147 patients would need to be enrolled to detect recurrence-free cure of $70 \%$ with $80 \%$ power and 5\% Type 1 error, assuming 15\% LTFU after treatment or per protocol withdrawal [14]. Study data were collected in standardised forms and recorded using Koch6 (MSF, Paris, France) and EpiInfo version 6 (CDC, Atlanta, GA, USA). Statistical analysis was conducted in Stata v15 (StataCorp, College Station, TX, USA). The cohort study is reported according to the STROBE statement (www.strobestatement.org; completed STROBE checklist in supporting information).

Baseline characteristics were described using frequencies and percentages for categorical variables, and medians and interquartile ranges (IQRs) for continuous variables. Descriptive analysis was performed using Chi-squared tests for categorical variables, and Wilcoxon rank-sum tests for continuous variables. Logistic regression was used to estimate adjusted odds ratios and p-values for potential predictors of unsuccessful outcome at 1 year following treatment completion, excluding patients without confirmed rifampicin resistance and fluoroquinolone sensitivity. Model construction used a manual forward step-wise approach. Age was included a priori in the final model, with additional variables added sequentially based on likelihood-ratio testing of model fit.

\section{Ethical approval}

The study was approved by the Ethics Review Boards of MSF and Uzbekistan. All adults included in the study provided written informed consent; for children $<16$ years old, written consent was obtained from their parent or guardian and the children also provided assent. 


\section{Results}

\section{Descriptive data}

Overall, 128 patients were included for the primary outcome analysis (figure 1); 67 patients were female (52.3\%); median age was 30.1 years (IQR 23.8-44.4); median BMI was $19.5 \mathrm{~kg} \cdot \mathrm{m}^{-2}$ (IQR 17.8-21.5), with $35.2 \%$ (45 out of 128 ) patients having a $\mathrm{BMI} \leqslant 18.5 \mathrm{~kg} \cdot \mathrm{m}^{-2}$. Diabetes mellitus was detected in $7 \%$ (9 out of 128 ) and no people living with HIV were identified on screening. Cavities on baseline chest radiography were found in $42 \%$ of patients (50 out of 119; table 1).

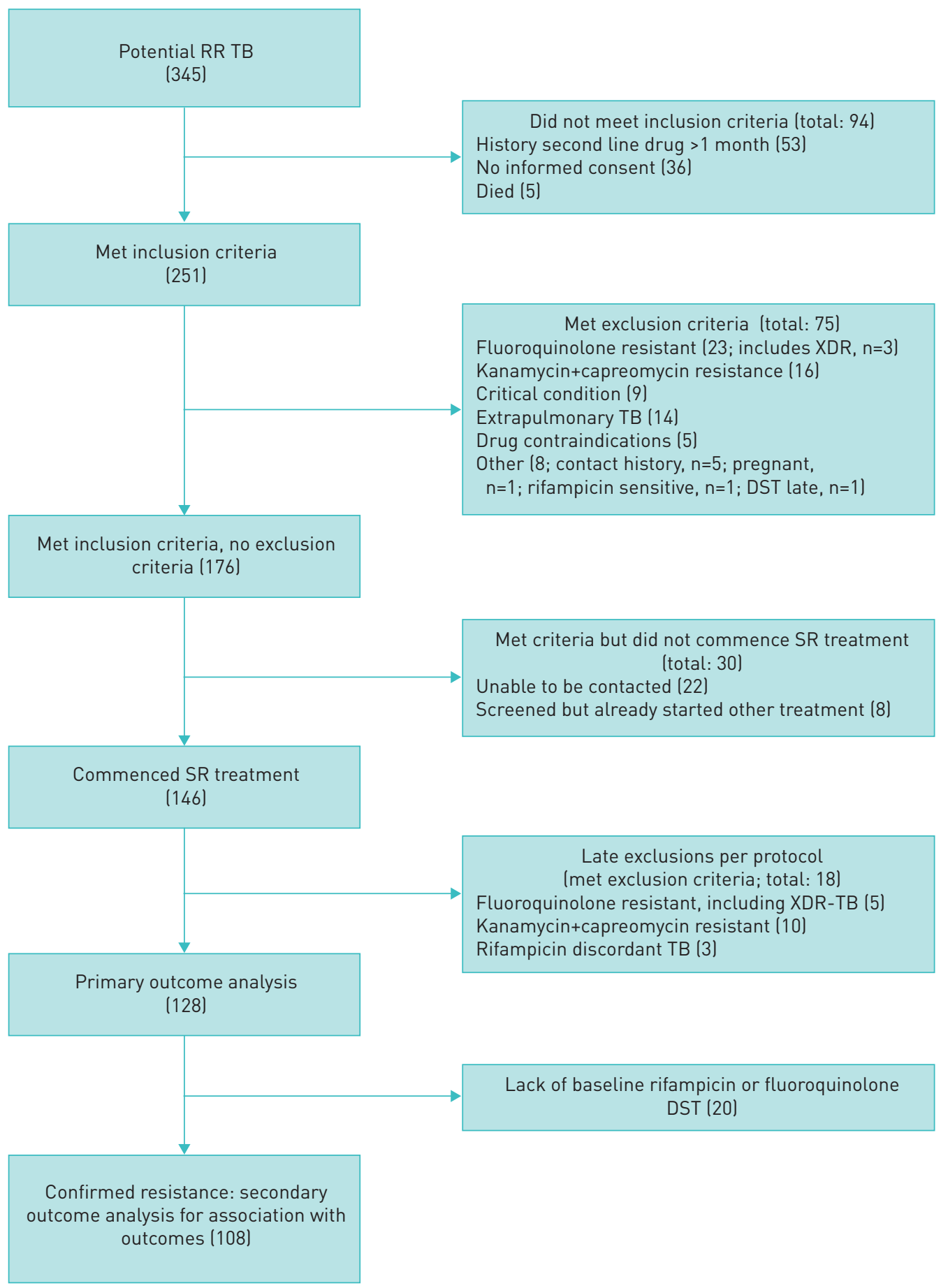

FIGURE 1 Patient enrolment flow diagram. RR: rifampicin-resistant; TB: tuberculosis; XDR: extensively drug-resistant; DST: drug susceptibility testing; SR: study regimen. 


\begin{tabular}{|c|c|c|c|}
\hline Characteristic & Total & Unsuccessful & Successful \\
\hline Total & $128(100 \%)$ & $41(32.0 \%)$ & 87 (68.0\%) \\
\hline Age years median (IQR) & $30.1(23.8-44.4)$ & $30.0(24.1-52.6)$ & $30.1(23.7-40.3)$ \\
\hline \multicolumn{4}{|l|}{ Age } \\
\hline$<15$ years & $2(1.6 \%)$ & $0(0.0 \%)$ & $2(2.3 \%)$ \\
\hline $15-44$ years & $95(74.2 \%)$ & $26(63.4 \%)$ & $69(79.3 \%)$ \\
\hline$\geqslant 45$ years & $31(24.2 \%)$ & $15(36.6 \%)$ & $16(18.4 \%)$ \\
\hline \multicolumn{4}{|l|}{ Sex } \\
\hline Male & $61(47.7 \%)$ & $19(46.3 \%)$ & $42(48.3 \%)$ \\
\hline Female & $67(52.3 \%)$ & $22(53.7 \%)$ & $45(51.7 \%)$ \\
\hline \multicolumn{4}{|l|}{ BMI } \\
\hline$>18.5 \mathrm{~kg} \cdot \mathrm{m}^{-2}$ & $83(64.8 \%)$ & 27 (65.9\%) & $56(64.4 \%)$ \\
\hline$\leqslant 18.5 \mathrm{~kg} \cdot \mathrm{m}^{-2}$ & 45 (35.2\%) & $14(34.1 \%)$ & $31(35.6 \%)$ \\
\hline \multicolumn{4}{|l|}{ Employment ${ }^{\#}$} \\
\hline Not employed & $110(89.4 \%)$ & $33(84.6 \%)$ & 77 (91.7\%) \\
\hline Employed & $13(10.6 \%)$ & $6(15.4 \%)$ & $7(8.3 \%)$ \\
\hline \multicolumn{4}{|l|}{ Diabetes mellitus } \\
\hline No & $119(93.0 \%)$ & $36(87.8 \%)$ & $83(95.4 \%)$ \\
\hline Yes & $9(7.0 \%)$ & $5(12.2 \%)$ & $4(4.6 \%)$ \\
\hline \multicolumn{4}{|l|}{ HIV status } \\
\hline Negative & $107(83.6 \%)$ & $32(78.0 \%)$ & 75 (86.2\%) \\
\hline Positive & 0 & 0 & 0 \\
\hline HIV status unknown & $21(16.4 \%)$ & $9(22.0 \%)$ & $12(13.8 \%)$ \\
\hline \multicolumn{4}{|l|}{ Previous TB treatment } \\
\hline New & $88(68.8 \%)$ & $29(70.7 \%)$ & 59 (67.8\%) \\
\hline Previously treated & $30(23.4 \%)$ & $11(26.8 \%)$ & $19(21.8 \%)$ \\
\hline \multicolumn{4}{|l|}{ Radiography findings \# } \\
\hline Noncavitary & $69(58.0 \%)$ & $20(52.6 \%)$ & $49(60.5 \%)$ \\
\hline Cavitary & $50(42.0 \%)$ & $18(47.4 \%)$ & 32 (39.5\%) \\
\hline \multicolumn{4}{|l|}{ Baseline smear status $\#$} \\
\hline Negative & $65(52.0 \%)$ & $17(42.5 \%)$ & $48(56.5 \%)$ \\
\hline Positive & $60(48.0 \%)$ & $23(57.5 \%)$ & $37(43.5 \%)$ \\
\hline \multicolumn{4}{|l|}{ Baseline culture status ${ }^{\#}$} \\
\hline Negative & $30(23.4 \%)$ & $8(19.5 \%)$ & $22(25.3 \%)$ \\
\hline Positive & $96(75.0 \%)$ & $32(78.0 \%)$ & $64(73.6 \%)$ \\
\hline \multicolumn{4}{|c|}{ Baseline pyrazinamide resistance } \\
\hline Sensitive & $23(27.7 \%)$ & $3(9.7 \%)$ & 20 (38.5\%) \\
\hline Resistant & $60(72.3 \%)$ & $28(90.3 \%)$ & $32(61.5 \%)$ \\
\hline Missing & 45 & 10 & 35 \\
\hline \multicolumn{4}{|c|}{ Baseline ethambutol resistance " } \\
\hline Sensitive & $28(32.9 \%)$ & $5(16.7 \%)$ & $23(41.8 \%)$ \\
\hline Resistant & $57(67.1 \%)$ & $25(83.3 \%)$ & $32(58.2 \%)$ \\
\hline Missing & 43 & 11 & 32 \\
\hline \multicolumn{4}{|c|}{ Baseline kanamycin resistance ${ }^{\pi}$} \\
\hline Sensitive & 75 (73.5\%) & $25(73.5 \%)$ & 50 (73.5\%) \\
\hline Resistant & $27(26.5 \%)$ & $9(26.5 \%)$ & $18(26.5 \%)$ \\
\hline Missing & 26 & 7 & 19 \\
\hline \multicolumn{4}{|c|}{ Baseline isoniazid-resistance mutations" } \\
\hline Resistant katG & $48(90.6 \%)$ & $15(93.8 \%)$ & $33(89.2 \%)$ \\
\hline Resistant inhA & $1(1.9 \%)$ & $0(0 \%)$ & $1(2.7 \%)$ \\
\hline Resistant katG+inhA & $4(7.5 \%)$ & $1(6.3 \%)$ & $3(8.1 \%)$ \\
\hline Missing & 75 & 25 & 50 \\
\hline \multicolumn{4}{|l|}{ Haemoglobin } \\
\hline$\geqslant 9.0 \mathrm{~g} \cdot \mathrm{dL}^{-1}$ & $57(44.5 \%)$ & $18(43.9 \%)$ & $39(44.8 \%)$ \\
\hline$<9.0 \mathrm{~g} \cdot \mathrm{dL}^{-1}$ & $71(55.5 \%)$ & $23(56.1 \%)$ & $48(55.2 \%)$ \\
\hline \multicolumn{4}{|l|}{ Glomerular filtration rate } \\
\hline$\geqslant 90 \mathrm{~mL} \cdot \mathrm{min}^{-1}$ & $108(84.4 \%)$ & $33(80.5 \%)$ & $75(86.2 \%)$ \\
\hline$<90 \mathrm{~mL} \cdot \mathrm{min}^{-1}$ & $20(15.6 \%)$ & $8(19.5 \%)$ & $12(13.8 \%)$ \\
\hline \multicolumn{4}{|l|}{ Initial injectable agent } \\
\hline Capreomycin & $110(85.9 \%)$ & $34(82.9 \%)$ & $76(87.4 \%)$ \\
\hline Kanamycin & $18(14.1 \%)$ & $7(17.1 \%)$ & $11(12.6 \%)$ \\
\hline
\end{tabular}




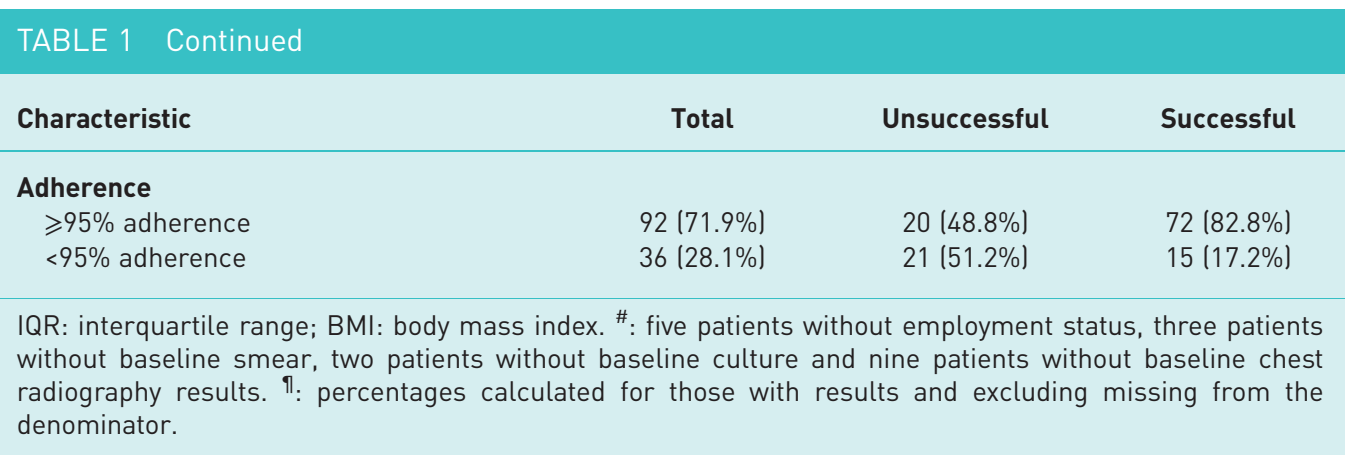

At baseline, $48.0 \%$ (60 out of 125) of patients were sputum-smear positive. Amongst those with baseline DST results, $72.3 \%$ (60 out of 83 ) exhibited pyrazinamide resistance, $67.0 \%$ (57 out of 85 ) had ethambutol resistance and 26.5\% (27 out of 102) had kanamycin resistance. Isoniazid-resistance mutations within kat $G$ were detected in $98.1 \%$ (52 out of 53) and inhA in 9.4\% (5 out of 53) of patients. A high proportion of baseline DST results had not been performed, were contaminated or not recorded during the specified period (table 1). Molecular and phenotypic fluoroquinolone DST results were unavailable for 20 patients (20 out of 128, 15.6\%), with only molecular DST results recorded for 5 additional patients (5 out of 128, 3.9\%).

\section{Outcomes}

At the end of treatment, $71.9 \%$ (92 out of 128) patients achieved a successful outcome, with $68 \%$ (87 out of 128) achieving 1-year recurrence-free cure. Unsuccessful outcomes 1-year after treatment were: treatment failure $17.2 \%$ (22 out of 128 ), LTFU 12.5\% (16 out of 128 ), death $1.5 \%$ (2 out of 128), recurrence $0.8 \%$ ( 1 out of 128 ) (table 2 ).

\section{Adverse events and amplification of resistance}

Adverse events were common, with 78.1\% (100 out of 128) patients reporting at least one adverse event, and a total of 826 adverse events in the cohort (table 3). Half of all patients (64 out of 128) had five or more adverse events. The majority $(94.4 \%, 780$ out of 826$)$ of adverse events were grade 1 and 2 . A total of 46 grade 3 or higher adverse events were experienced by $28(21.9 \%)$ patients. There were 15 serious adverse events (SAEs) reported from $14(10.9 \%)$ patients (table 3). Eight SAEs were assessed as being possibly related to the drug regimen, with seven SAEs assessed as unlikely related. Amongst patients who

TABLE 2 Treatment outcomes at end of treatment and after 1-year follow-up among patients treated with a standardised shorter multidrug-resistant tuberculosis regimen

Outcome evaluated at end of treatment $(n=128)$
Outcome evaluated 1 year after treatment completion $(n=128)$

\begin{tabular}{|c|c|c|}
\hline \multicolumn{3}{|l|}{ Successful outcomes } \\
\hline Cure & $55(43.0 \%)$ & \\
\hline Complete & $37(28.9 \%)$ & \\
\hline Recurrence-free cure & & $87(68.0 \%)$ \\
\hline \multicolumn{3}{|l|}{ Unsuccessful outcomes } \\
\hline Died & $2(1.5 \%)$ & $2(1.5 \%)$ \\
\hline $\begin{array}{l}\text { Treatment regimen failure, } \\
\text { microbiological }\end{array}$ & $16(12.5 \%)$ & $16(12.5 \%)$ \\
\hline $\begin{array}{l}\text { Treatment regimen failure, } \\
\text { adverse event }\end{array}$ & $6(4.7 \%)$ & $6(4.7 \%)$ \\
\hline LTFU during treatment & $12(9.4 \%)$ & $12(9.4 \%)$ \\
\hline LTFU after treatment & & $4(3.1 \%)^{\#}$ \\
\hline Recurrence & & $1(0.8 \%)^{\pi}$ \\
\hline
\end{tabular}

LTFU: lost to follow-up. ${ }^{\#}: 4.3 \%$ (four out of 92) patients who successfully completed treatment were LTFU after treatment completion; ": overall, one $(0.8 \%)$ patient had recurrence, for a 1 -year recurrence rate of 1.1 events per 100 patient-years. 


\begin{tabular}{|c|c|c|c|c|c|}
\hline & Grade $1^{\#}$ & Grade $2^{\#}$ & Grade $3^{\#}$ & Grade $4^{\#}$ & Total" \\
\hline Nausea and vomiting & $158(81.0 \%)$ & $34(17.4 \%)$ & $3(1.5 \%)$ & 0 & $195(23.6 \%)$ \\
\hline Weakness, fatigue & $59(67.8 \%)$ & $22(25.3 \%)$ & $6(6.9 \%)$ & 0 & $87(10.5 \%)$ \\
\hline Abdominal pain & $75(86.2 \%)$ & $9(10.3 \%)$ & $2(2.2 \%)^{+}$ & $1(1.1 \%)^{+}$ & $87(10.5 \%)$ \\
\hline Headache & $56(83.6 \%)$ & $10(14.9 \%)$ & $1(1.5 \%)$ & 0 & $67(8.1 \%)$ \\
\hline Arthralgia & $51(85.0 \%)$ & $7(11.7 \%)$ & $2(3.3 \%)^{+}$ & 0 & $60(7.3 \%)$ \\
\hline Renal failure & $36(64.3 \%)$ & $14(25.0 \%)$ & $6(10.7 \%)$ & 0 & $56(6.8 \%)$ \\
\hline Anorexia/constipation & $36(67.9 \%)$ & $16(30.2 \%)$ & $1(1.9 \%)$ & 0 & $53(6.4 \%)$ \\
\hline Ototoxicity & $30(75 \%)$ & $7(17.5 \%)$ & $3(7.5 \%)^{+}$ & 0 & $40(4.8 \%)$ \\
\hline Diarrhoea & $22(78.6 \%)$ & $6(21.4 \%)$ & 0 & 0 & $28(3.4 \%)$ \\
\hline Flushing/itching & $20(83.3 \%)$ & $4(16.7 \%)$ & 0 & 0 & $24(2.9 \%)$ \\
\hline Hepatitis & $17(81.0 \%)$ & $3(14.3 \%)^{+}$ & $1(4.8 \%)$ & 0 & $21(2.5 \%)$ \\
\hline Rash & $15(75.0 \%)$ & $3(15.0 \%)$ & $2(10.0 \%)$ & 0 & $20(2.4 \%)$ \\
\hline QTc prolongation & $6(40.0 \%)$ & $4(26.7 \%)$ & $4(26.7 \%)$ & $1(6.7 \%)$ & $15(1.8 \%)$ \\
\hline Anaemia & $6(50.0 \%)$ & $3(25.0 \%)$ & $3(25.0 \%)$ & 0 & $12(1.5 \%)$ \\
\hline Depression/anxiety & $7(63.6 \%)$ & $1(9.1 \%)$ & $2(19.2 \%)$ & $1(9.1 \%)$ & $11(1.3 \%)$ \\
\hline Electrolyte loss & $9(100.0 \%)$ & 0 & 0 & 0 & $9(1.1 \%)$ \\
\hline Neuromuscular weakness & $5(83.3 \%)$ & 0 & $1(16.7 \%)^{+}$ & 0 & $6(0.7 \%)$ \\
\hline Visual loss & $3(75.0 \%)$ & $1(25.0 \%)$ & 0 & 0 & $4(0.5 \%)$ \\
\hline Cramps & $2(50.0 \%)$ & $2(50.0 \%)$ & 0 & 0 & $4(0.5 \%)$ \\
\hline Gastritis & $3(75.0 \%)$ & $1(25.0 \%)$ & 0 & 0 & $4(0.5 \%)$ \\
\hline Altered mental status & $2(50.0 \%)$ & 0 & $1(25.0 \%)^{+}$ & $1(25.0 \%)^{+}$ & $4(0.5 \%)$ \\
\hline Skin colour change & $2(66.7 \%)$ & $1(33.3 \%)$ & 0 & 0 & $3(0.4 \%)$ \\
\hline Hypothyroidism & 0 & $3(2.0 \%)$ & 0 & 0 & $3(0.4 \%)$ \\
\hline Psychosis & $3(100.0 \%)$ & 0 & 0 & 0 & $3(0.4 \%)$ \\
\hline Allergic reaction & $1(50.0 \%)$ & $1(50.0 \%)^{+}$ & 0 & 0 & $2(0.2 \%)$ \\
\hline Haemorrhage & $1(50.0 \%)^{+}$ & 0 & 0 & $1(50.0 \%)^{+}$ & $2(0.2 \%)$ \\
\hline Infection & $1(50.0 \%)$ & 0 & $1(50.0 \%)^{+}$ & 0 & $2(0.2 \%)$ \\
\hline Seizure & $1(100.0 \%)$ & 0 & 0 & 0 & $1(0.1 \%)$ \\
\hline Syncope & 0 & $1(100.0 \%)^{+}$ & 0 & 0 & $1(0.1 \%)$ \\
\hline Hypoglycaemia & 0 & 0 & $1(100.0 \%)^{+}$ & 0 & $1(0.1 \%)$ \\
\hline Diabetes mellitus & 0 & 0 & 0 & $1(100.0 \%)^{+}$ & $1(0.1 \%)$ \\
\hline Total & $627(75.9 \%)$ & 153 (18.5\%) & $40(4.8 \%)$ & $6(0.7 \%)$ & $826(100 \%)$ \\
\hline
\end{tabular}

completed treatment, no replacement outside the same drug class occurred, and only one patient permanently ceased a drug (prothionamide) during the continuation phase.

Amongst 22 patients who suffered treatment failure, 16 (72.7\%) had confirmed microbiological failure. Resistance amplification to injectables or fluoroquinolones was confirmed in eight (36.4\%) patients: seven acquired resistance to ofloxacin, and one acquired resistance to both ofloxacin and capreomycin. The single patient with recurrence had confirmed MDR-TB, but not confirmed amplification of fluoroquinolone resistance.

\section{Predictors of unsuccessful outcome}

Factors associated with unsuccessful outcome at 1 year following treatment completion in univariable analysis included pyrazinamide resistance (OR 6.02; 95\% CI 1.61; 22.47), ethambutol resistance (OR 3.44; 95\% CI 1.14 ; 10.36), resistance to both pyrazinamide and ethambutol (OR 4.83 ; 95\% CI $1.77 ; 13.18$ ) and adherence less than 95\% (OR 3.96; 95\% CI 1.57; 9.97). Strong evidence of an association between baseline resistance to both pyrazinamide and ethambutol, and adherence $<95 \%$ remained following adjustment for age (table 4).

\section{Outcomes of late exclusions}

18 patients met exclusion criteria after having commenced the SSR: 5 were fluoroquinolone-resistant, 10 were dual-injectable-resistant, and 3 were rifampicin-sensitive (table 5) detected on phenotypic DST. These patients were switched to individualised treatment according to programme guidelines. Thirteen (72.2\%) patients achieved a successful outcome following treatment with a median duration of 20 months 
TABLE 4 Comparison of patient characteristics with 1-year post-completion treatment response amongst all patients with confirmed baseline ofloxacin drug-susceptibility testing (108 patients)

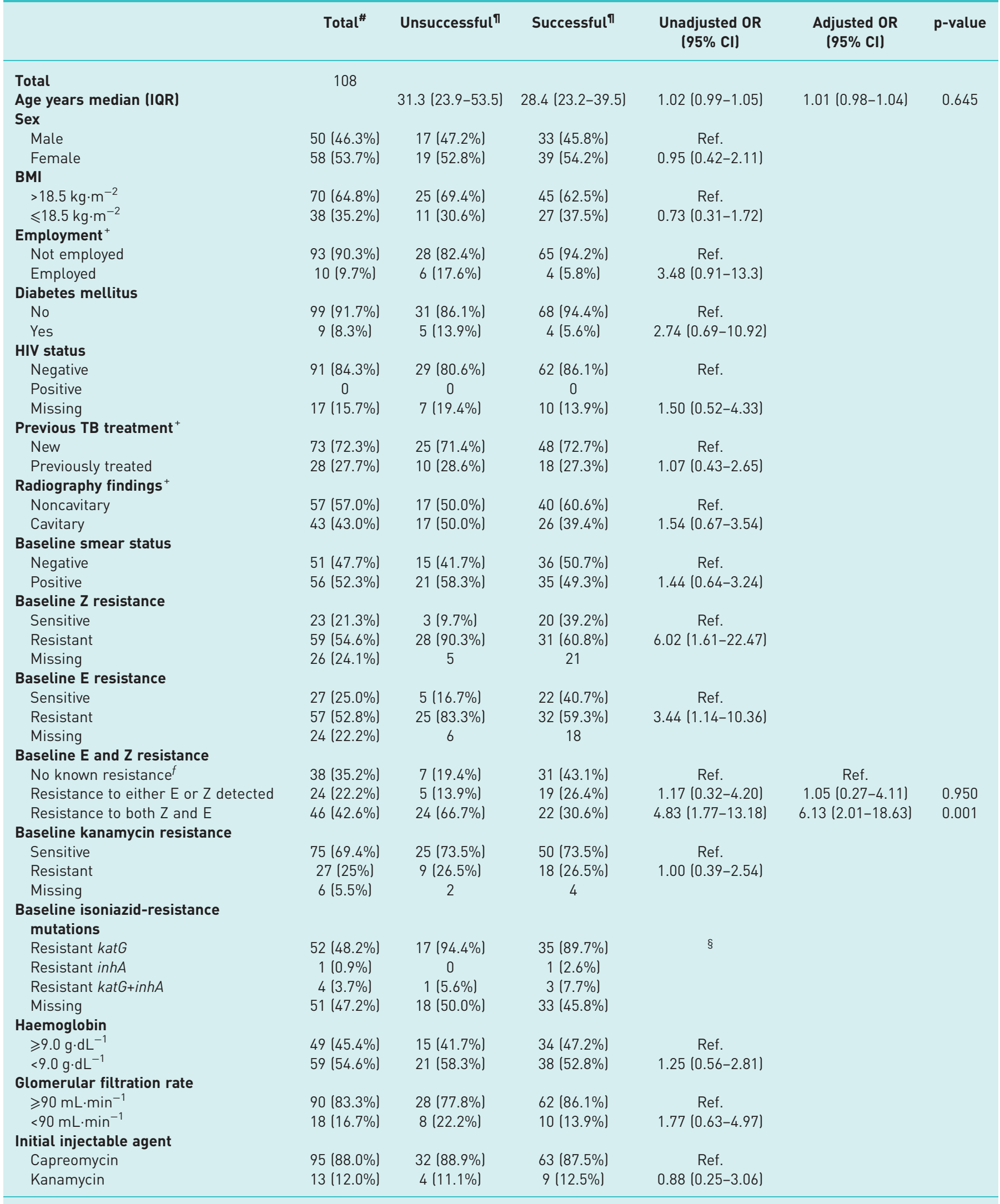


TABLE 4 Continued

Total $^{\# \quad \text { Unsuccessful" Successful }}$

Adjusted OR (95\% Cl)

\section{Adherence}

$\geqslant 95 \%$ adherence

79 (73.2\%)

$19(52.8 \%)$

$29(26.8 \%)$

$17(47.2 \%)$

$60(83.3 \%)$

$12(16.7 \%)$

Ref.
$3.96(1.57-9.97)$

Ref.

$5.33(1.73-16.36)$

20 patients from the original 128 were excluded from univariable and multivariable analysis due to a lack of rifampicin and ofloxacin drug-susceptibility testing within 90 days prior to 7 days after commencement of standardised shorter regimen. Of the 108 included patients, 103 were ofloxacin-sensitive by baseline ofloxacin culture and five were sensitive by baseline Hain SL. IQR: interquartile range; BMI: body

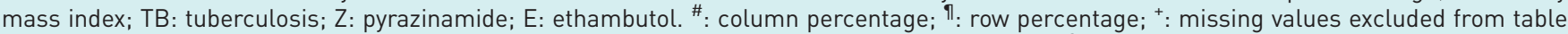
as $<10 \%$ of values (employment, $n=5$; previous TB treatment, $n=7$ ); radiography findings, $n=8$ ); ${ }^{\S}$; not included in logistic regression as high proportion missing values and overwhelming majority katG mutation; ${ }^{f}$ : includes sensitive or missing results to $E$ and $Z$.

(IQR 7.5-21.5). Four patients were continued on SSR, due to patient and clinician choice in discussion with the principal investigator, and achieved a successful outcome.

\section{Discussion}

In this cohort study we found that a SSR with standard-dose moxifloxacin achieved moderate success with a low recurrence rate. We showed lower successful outcomes than have been reported from other settings, with high treatment failure due to both microbiological failure with amplification of fluoroquinolone resistance, and regimen toxicity $[3,4,22]$. Diminished adherence and resistance to both pyrazinamide and ethambutol were associated with unsuccessful outcome.

Previously published studies of similar SSR regimens have reported end-of-treatment success between 81.6-89.2\% [3, 22-25]. There are several potential reasons for the discrepancy between these rates and our outcomes. In our study, patients were started on capreomycin until injectable sensitivity results were known. Recently, inclusion of capreomycin in RR/MDR-TB regimens has been negatively associated with treatment response [26]. The regimen included moxifloxacin at a standard dosing of $400 \mathrm{mg}$ daily, while fluoroquinolones were used at up to twice this dosage in other studies [3,4]. It is unclear whether switching to amikacin and higher-dose moxifloxacin would improve outcomes in this region given uncertainty about circulating eis, gyrA and $g y r B$ mutations. In addition, the SSR included prothionamide throughout the treatment period. We did not have phenotypic prothionamide DST available; however, only a minority of those tested had inhA mutations.

Treatment failure was $17.1 \%$, which is higher than internationally reported pooled rates of $11 \%(10-12 \%)$ for the longer MDR-TB regimen excluding extensively drug-resistant TB [2]. The majority of treatment failure was microbiologically confirmed, while more than a quarter was due to toxicity or intolerance. LTFU with the long MDR-TB regimen has been reported to be $20 \%$ within the Karakalpakstan MDR-TB program, higher than the $9.4 \%$ LTFU rate in our study cohort [12]. LTFU may have been reduced further if prothionamide was not used for the full duration as in other cohorts [3,22]. Our results support the STREAM trial outcomes, suggesting that while a SSR may considerably reduce LTFU, these regimens also have a higher rate of treatment failure [4]. It is concerning that more than one-third of patients with treatment failure had confirmed amplification of resistance mutations to ofloxacin.

Recently, the WHO has announced changes to the treatment of drug-resistant TB, recommending shorter, all-oral, bedaquiline-containing regimens in place of the injectable-containing SSR [27]. In the primary analysis on which the WHO guidelines decision was based, treatment success rates for the all-oral bedaquiline-containing regimen was $73 \%$ versus $60 \%$ in the SSR [28]. Uncertainty exists over whether those with isolates resistant to ethambutol, pyrazinamide or ethionamide should be excluded from SSR treatment, particularly in the absence of an accurate rapid diagnostic test for these drugs, or treated with alternative medications $[9,11]$. The design of the seven-drug "Bangladesh" SSR regimen was such that resistance to one or two noncore drugs would still leave sufficient likely effective drugs to achieve a cure [29]. In a large meta-analysis, fluoroquinolone and pyrazinamide resistance was associated with lower treatment success [23]. In a large study in nine countries in Africa, there was no association found between pyrazinamide and ethambutol resistance and unsuccessful outcomes, likely due to the very small number of patients that failed [22]. Our study looked at combined resistance of pyrazinamide and ethambutol, which was strongly associated with unsuccessful outcomes at 1 year following treatment completion, even when accounting for other measured confounders. Number of effective drugs within a regimen has been associated with RR/MDR-TB treatment outcomes, with inclusion of drugs with 
TABLE 5 Characteristics, management and outcomes of late exclusions from the standardised shorter multidrug-resistant (MDR) tuberculosis regimen

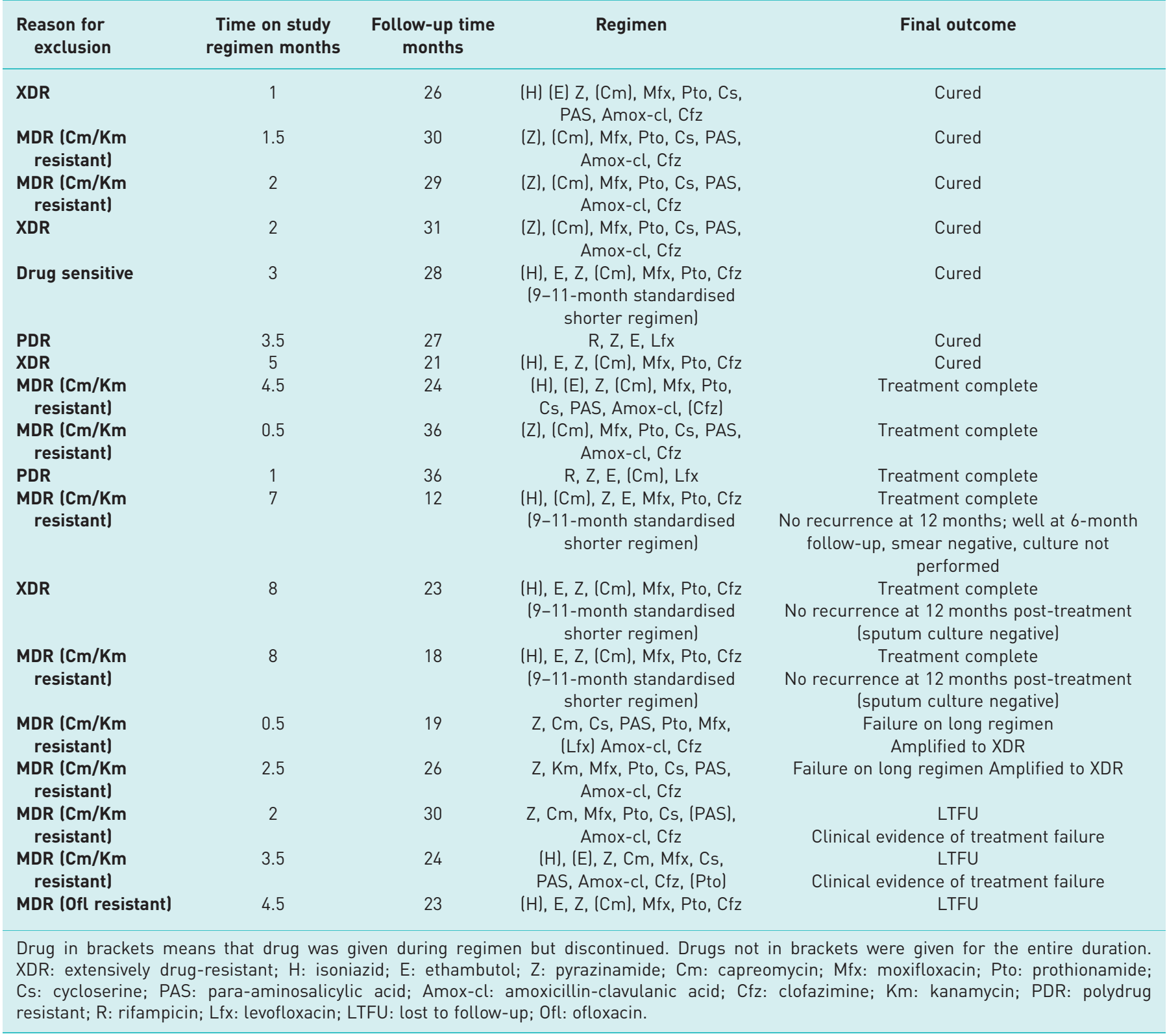

resistance or without DST potentially adding cost and toxicity [30]. Given the high rates globally of pyrazinamide, ethambutol and ethionamide resistance, this raises questions of continued inclusion of these drugs in standardised shorter regimens in the absence of DST-confirmed susceptibility.

Patients who missed more than $5 \%$ of prescribed doses had greater odds of unsuccessful outcomes. The programme in Uzbekistan provided counselling and psychosocial support to patients within a decentralised system. Even with DOT, patients can still miss doses due to medication adverse events, social marginalisation, stigma, and economic consequences [31,32]. DOT can ignore the patient's perspective, and fail to provide required support for adherence [33]. Empowerment of patients through provision of knowledge and greater control over treatment are factors that have been shown to positively influence adherence in Uzbekistan [34]. The high proportion of patients experiencing adverse events in our study may also have impacted on treatment adherence and interruptions. Programmatic implementation of shorter regimens requires close attention to the management of side effects, and infrastructure to support patient adherence. Including adherence data is important for future short-regimen studies, given our findings. 
There are a number of strengths of this study. Firstly, this study was performed under programmatic conditions and therefore reflects a realistic scenario; results are likely to be generalisable to programs in similar settings. Secondly, there was careful attention to monitoring with the majority of patients having baseline DST results, attention to standardised reporting of adverse events, and follow-up for potential relapse for 1 year after treatment completion. Finally, the study has been able to provide important new data on the importance of ethambutol and pyrazinamide resistance and the impact of treatment adherence on risk of failure.

There were a number of limitations in this single-arm study. A proportion of patients did not have baseline rifampicin and pyrazinamide resistance results within 3 months of treatment beginning. Inclusion of patients with a long delay between baseline test performance and the start of treatment may have introduced survival bias, resulting in overestimation of treatment effectiveness. Additionally, partial treatment and development of undetected resistance may have resulted in over- or under-estimation of regimen performance. Missing baseline DST results may have underestimated the influence of pyrazinamide and ethambutol resistance status on treatment response. Due to missing DST results, patients with unidentified fluoroquinolone resistance may still have been included in the study. The MTBDRsl version 1 has a moderate sensitivity of $83-85 \%$, with five patients (5 out of 146, 3.4\%) enrolled subsequently shown to have fluoroquinolone resistance [35]. We therefore only included patients with DST-confirmed fluoroquinolone susceptibility in the univariable and multivariable analysis. Phenotypic DST for ethambutol, pyrazinamide and capreomycin is recognised as unreliable and, therefore, may have resulted in misclassification bias. Similarly, use of ofloxacin for fluoroquinolone DST may not have accurately assessed moxifloxacin susceptibility. Next-generation sequencing would have reduced this bias but was unavailable. Finally, the limited cohort size yields imprecision during multivariable analysis.

Updated WHO guidelines for RR/MDR-TB conditionally recommend a shorter bedaquiline-containing regimen for patients without previous exposure to second-line treatment and without fluoroquinolone resistance $[25,28]$. Outcomes of substituting bedaquiline for the injectable agent when toxicity occurred are promising [36]. Further operational research using SSRs incorporating more efficacious drugs, including bedaquiline and linezolid, and removing drugs with high rates of resistance globally, including ethionamide, pyrazinamide and ethambutol, would yield important real-world results, while waiting for ongoing randomised trials to finish [37].

\section{Conclusions}

Overall treatment success of the studied SSR was moderate, with high rates of microbiological failure and amplification of fluoroquinolone resistance, as compared with other studies; the reasons for these differences are unclear. Baseline drug resistance and small reductions in adherence were associated with unsuccessful outcomes, highlighting the importance of availability of DST for drugs used within a SSR and reinforcing the importance of supporting adherence. Our results argue for the urgent need for improved SSRs in order to effectively respond to RR/MDR-TB in settings with high levels of first and second-line drug resistance.

Acknowledgements: The authors wish to thank the data safety monitoring board members (Kai Blondal, Colleen Acosta and Andrei Dadu) and all of the Uzbekistan TB programme staff and MSF staff who support TB patient care in Karakalpakstan. Emma Veitch, freelance medical editor for MSF UK, provided editorial assistance and her work was funded by MSF UK.

Support statement: This study was funded by MSF, Operational Centre Amsterdam.

Conflict of interest: P. du Cros reports other funded work from TB Alliance for introduction of pretomanid, outside the submitted work. A. Khamraev has nothing to disclose. Z. Tigay has nothing to disclose. T. Abdrasuliev has nothing to disclose. J. Greig has nothing to disclose. G. Cooke has nothing to disclose. K. Herboczek has nothing to disclose. T. Pylypenko has nothing to disclose. C. Berry has nothing to disclose. A. Ronnachit has nothing to disclose. D. Lister has nothing to disclose. S. Dietrich has nothing to disclose. C. Ariti has nothing to disclose. K. Safaev has nothing to disclose. B-T. Nyang'wa has nothing to disclose. N. Parpieva has nothing to disclose. M. Tillashaykhov has nothing to disclose. J. Achar has nothing to disclose.

\section{References}

1 World Health Organization. Global Tuberculosis Report 2019. Geneva, World Health Organization, 2019.

2 Bastos ML, Lan Z, Menzies D. An updated systematic review and meta-analysis for treatment of multidrug-resistant tuberculosis. Eur Respir J 2017; 49: 1600803.

3 van Deun A, Maug A, Salim MAH, et al. Short, highly effective, and inexpensive standardized treatment of multidrug-resistant tuberculosis. Am J Respir Crit Care Med 2010; 182: 684-692.

4 Nunn AJ, Phillips PPJ, Meredith SK, et al. A trial of a shorter regimen for rifampin-resistant tuberculosis. $N$ Engl J Med 2019; 380: 1201-1213.

5 Falzon D, Schünemann HJ, Harausz E, et al. World Health Organization treatment guidelines for drug-resistant tuberculosis, 2016 update. Eur Respir J 2017; 49: 1602308. 
6 World Health Organization. WHO Consolidated Guidelines on Drug-resistant Tuberculosis Treatment. Geneva, World Health Organization, 2019.

7 Guglielmetti L, Varaine F, Huerga $\mathrm{H}$, et al. Shortened multidrug-resistant tuberculosis treatment in settings with a high prevalence of ofloxacin resistance. Eur Respir J 2017; 50: 1700598.

8 Lange C, Duarte R, Fréchet-Jachym M, et al. Limited benefit of the new shorter multidrug-resistant tuberculosis regimen in Europe. Am J Respir Crit Care Med 2016; 194: 1029-1031.

9 Sotgiu G, Tiberi S, D’Ambrosio L, et al. WHO recommendations on shorter treatment of multidrug-resistant tuberculosis. Lancet 2016; 387: 2486-2487.

10 Berry C, Achar J, du Cros P. WHO recommendations for multidrug-resistant tuberculosis. Lancet 2016; 388: 2234.

11 Varaine F, Guglielmetti L, Huerga $\mathrm{H}$, et al. Eligibility for the shorter multidrug-resistant tuberculosis regimen: ambiguities in the World Health Organization recommendations. Am J Respir Crit Care Med 2016; 194: 1028-1029.

12 Lalor MK, Greig J, Allamuratova S, et al. Risk factors associated with default from multi- and extensively drug-resistant tuberculosis treatment, Uzbekistan: a retrospective cohort analysis. PLoS ONE 2013; 8: e78364.

13 World Health Organization. Treatment of Drug-resistant TB: the use of Short Regimens. www.who.int/tb/areas-ofwork/drug-resistant-tb/treatment/short-regimens/en/ Date last accessed: July 21, 2019. Date last updated: November 19, 2015.

14 du Cros P, Khamraev A, Tillashaikhov M, et al. Research Protocol: Effectiveness of a Simplified Short Regimen for Multidrug Resistant Tuberculosis Treatment in Karakalpakstan, Uzbekistan. https://fieldresearch.msf.org/handle/ 10144/322296 Date last accessed: July 21, 2019. Date last updated July 01, 2014.

15 Cockcroft DW, Gault H. Prediction of creatinine clearance from serum creatinine. Nephron 1976; 16: 31-41.

16 Moss AJ. Introductory note to a translated classic article of L. S. Fridericia. Ann Noninvasive Electrocardiol 2003 8: $341-342$.

17 Fridericia LS. The duration of systole in an electrocardiogram in normal humans and in patients with heart disease. Ann Noninvasive Electrocardiol 2003; 8: 343-351.

18 US Department of Health and Human Services, National Institutes of Health, National Institute of Allergy and Infectious Diseases, Division of AIDS. Division of AIDS Table for Grading the Severity of Adult and Pediatric Adverse Events, Version 1.0. www.rsc.niaid.nih.gov/sites/default/files/table-for-grading-severity-of-adult-pediatricadverse-events.pdf Date last accessed: July 21, 2019. Date last updated: August 2009.

19 Rüsch-Gerdes S, Pfyffer GE, Casal M, et al. Multicenter laboratory validation of the BACTEC MGIT 960 technique for testing susceptibilities of Mycobacterium tuberculosis to classical second-line drugs and newer antimicrobials. J Clin Microbiol 2006; 44: 688-692.

20 World Health Organization STOP TB Department. Policy Guidance on Drug-susceptibility Testing (DST) of Second-line Antituberculosis Drugs. www.who.int/publications/i/item/WHO-HTM-TB-2008.392 Date last accessed: October 10, 2020. Date last updated: 2008.

21 World Health Organization. Definitions and Reporting Framework for Tuberculosis 2013 Revision Updated December 2014. Geneva, World Health Organisation, 2014.

22 Trébucq A, Schwoebel V, Kashongwe Z, et al. Treatment outcome with a short multidrug-resistant tuberculosis regimen in nine African countries. Int J Tuberc Lung Dis 2018; 22: 17-25.

23 Khan FA, Salim MAH, du Cros P, et al. Effectiveness and safety of standardised shorter regimens for multidrug-resistant tuberculosis: individual patient data and aggregate data meta-analyses. Eur Respir J 2017; 50 1700061

24 Kuaban C, Noeske J, Rieder HL, et al. High effectiveness of a 12-month regimen for MDR-TB patients in Cameroon. Int J Tuberc Lung Dis 2015; 19: 517-524.

25 Piubello A, Harouna SH, Souleymane MB, et al. High cure rate with standardised short-course multidrugresistant tuberculosis treatment in Niger: no relapses. Int J Tuberc Lung Dis 2014; 18: 1188-1194.

26 Ahmad N, Ahuja SD, Akkerman OW, et al. Treatment correlates of successful outcomes in pulmonary multidrug-resistant tuberculosis: an individual patient data meta-analysis. Lancet 2018; 392: 821-834.

27 World Health Organization. Rapid Communication: Key Changes to Treatment of Drug-resistant Tuberculosis. Geneva, World Health Organization, 2019.

28 World Health Organization. WHO Consolidated Guidelines on Tuberculosis. Module 4. www.ncbi.nlm.nih.gov/ books/NBK558570 Date last accessed: 20 September, 2020. Date last updated: 2020.

29 Deun AV, Decroo T, Piubello A, et al. Principles for constructing a tuberculosis treatment regimen: the role and definition of core and companion drugs. Int J Tuberc Lung Dis; 22: 239-245.

30 Cegielski JP, Kurbatova E, van der Walt M, et al. Multidrug-resistant tuberculosis treatment outcomes in relation to treatment and initial versus acquired second-line drug resistance. Clin Infect Dis 2016; 62: 418-430.

31 Daftary A, Padayatchi N, O’Donnell M. Preferential adherence to antiretroviral therapy over tuberculosis treatment: A qualitative study of drug-resistant TB/HIV co-infected patients in South Africa. Glob Public Health 2014; 9: 1107-1116.

32 Brust JCM, Shah NS, van der Merwe TL, et al. Adverse events in an integrated, home-based treatment program for MDR-TB and HIV in KwaZulu-Natal, South Africa. J Acquir Immune Defic Syndr 2013; 62: 436-440.

33 Yin J, Wang X, Zhou L, et al. The relationship between social support, treatment interruption and treatment outcome in patients with multidrug-resistant tuberculosis in China: a mixed-methods study. Trop Med Int Health 2018; 23: 668-677.

34 Horter S, Stringer B, Greig J, et al. Where there is hope: a qualitative study examining patients' adherence to multi-drug resistant tuberculosis treatment in Karakalpakstan, Uzbekistan. BMC Infect Dis 2016; 16: 362.

35 Theron G, Peter J, Richardson M, et al. The diagnostic accuracy of the GenoType ${ }^{\circ}$ MTBDRsl assay for the detection of resistance to second-line anti-tuberculosis drugs. Cochrane Database Syst Rev 2014; CD010705.

36 Bastard M, Molfino L, Mutaquiha C, et al. Treatment outcomes of patients switching from an injectable drug to bedaquiline during short standardized treatment for multidrug-resistant tuberculosis in Mozambique. Clin Infect Dis 2019; 69: 1809-1811.

37 Seung KJ, Hewison C. Now is the time for shorter all-oral regimens for multidrug-resistant tuberculosis. Lancet Glob Health 2019; 7: e706 